\title{
Zusammenfassung und Schlussresümee
}

Das Poly-Werk verkörpert den Anspruch, neue Formen zu finden, die die zeitgleiche Vereinbarung verschiedener Stränge im selben Medium ermöglichen, ohne zwingend auf eine Synthese, eine Einheit zu zielen. Wie kaum eine andere Form in der Musik greifen Poly-Werke die Teilbarkeit und Flexibilität der Kreativität und der Aufmerksamkeit auf. Beide sind durchaus in der Lage, sich verschiedener linearer Verläufe bewusst zu sein, sie sowohl zu erdenken als auch zu verfolgen, sie zu bündeln oder zu selektieren. Die mehrfachen Möglichkeiten der sowohl einzelnen als auch - in mindestens einer Konstellation - simultanen Anordnung erzeugen kompositorisch Mehrdeutigkeit und Ambiguität.

Obgleich Poly-Werke sich wandelnde Wahrnehmungsweisen aufgreifen und das Werkobjekt verschiedenen Perspektiven aussetzen, erfolgte ihre Entwicklung in den vergangenen siebzig Jahren vergleichsweise unbemerkt vom Neue-Musik-Diskurs. Über den gesamten Zeitraum gesehen, treten sie zwar zunehmend mit einer gewissen Konstanz auf, jedoch niemals geballt. Ihre Geschichte setzt sich vor allem aus vereinzelten und voneinander unabhängigen Kompositionsansätzen zusammen, die auf den ersten Blick ein uneinheitliches, zersplittertes, vielfältiges Bild abgeben. Eine zeitlich progressive Entwicklung des Poly-Werks gibt es nicht. Ihre Verortung stützt sich deswegen vor allem auf die individuellen Ansätze, die die formalen Gestaltungsmöglichkeiten in verschiedene Richtungen leiteten. Dies machte eine Darstellung nach systematischen Kriterien erforderlich. Im Zentrum der vorliegenden Arbeit stehen Analysen ausgewählter Werke, die das kaleidoskopartige Spektrum der Möglichkeiten sichtbar machen und dieses nach bestimmten formalen Gesichtspunkten auch zu strukturieren versuchen. Bereits durch die Gruppierung der Werke zeigen sich latente und unterschwellige Verbindungen und Bezüge, die einen Vergleich auf der formalen Ebene ermöglichen. Neben dieser systematischen Erfassung war es ein wesentliches Ziel, die werkästhetischen Vorbedingungen und künstlerischen Prozesse zu reflektieren, aus denen das Poly-Werk hervorging.

Die Kernthese der Untersuchung war, dass das Poly-Werk den Werkgedanken zwar einerseits wahrt, ihn aber andererseits öffnet und die Einsheit des traditionellen Werkkonzepts relativiert und neu befragt. Ebenso wie die sogenannten „offenen Formen“ verkörpert das Poly-Werk ein relativistisches Denkmodell, das der Eindeutigkeit und Abgeschlossenheit des Werkkonzepts 
die Ambivalenz des Sowohl-als-auch an die Seite stellt. Um die für das PolyWerk spezifische Relativierung des Werkkonzepts zu verstehen und einzuordnen war es nötig, den Bedeutungshorizont des Begriffes „Offenheit“ aufzuschlüsseln. Auf diese Weise konnte eine Abgrenzung zu anderen offenen Werkkonzepten vorgenommen werden.

Die komplementäre Zusammensetzung verschiedener Teilwerke im PolyWerk führt zu einer der „offenen Form“ ähnlichen konstellativen Werkentität, die wie diese mehrere formale Möglichkeiten bietet, das Werk aufzuführen. Sowohl bei der "offenen Form“ als auch bei einem simultan aufgeführten "Circus" steht tendenziell eher die Quantität, die möglichst hohe Vielzahl an austauschbaren Möglichkeiten im Vordergrund. „Offene Formen“ betonen das Unverbindliche, das Nichtfestgelegte, das Streben nach „Unendlichkeit“. Dabei birgt eine Indifferenz der Möglichkeiten - ebenso wie die klare Eindeutigkeit die Gefahr, Mehrdeutigkeit und Ambiguität zu verhindern. Hier liegt meines Erachtens die entscheidende Weggabelung zwischen dem künstlerischen Ansatz von John Cage und der Theoriebildung von Umberto Eco. Cage legte es nicht darauf an, Bedeutung herbeizuführen. Seine Stücke sollten möglichst bedeutungsimmun sein. Dagegen interpretiert Eco die „offene Form“ so, dass sie durch eine Vielzahl an Möglichkeiten einen Bedeutungsüberschuss erzeugt. Durch die Unterscheidung zwischen Unbestimmtheit und Überbestimmtheit weist sein Konzept der „offenen Form“ in der Rückschau in vielerlei Hinsicht auf die Möglichkeiten des Poly-Werks treffender hin als auf die offenen Formen der Musik seiner Zeit.

Während die „offene Form“ ins „Unendliche“ weist, ein simultan aufgeführter „Circus" beliebig oder spontan entstehen kann, sind die Stränge im Poly-Werk idealerweise ausformuliert und in sich konsistent. Die Öffnung im Poly-Werk ist nicht unbegrenzt und damit auch nicht indifferent. Es handelt sich um eine "gebundene“ Öffnung, die sich sinnhaft auf einzelne konkrete andere Werkstränge bezieht, die auch konträre Eigenschaften besitzen können. Die Unverbindlichkeit von Beziehung wird in eine Exklusivität von Beziehung überführt. Im Gegensatz zur Indifferenz wird die Differenz zum Anderen hier explizit anerkannt und gesucht und ebenso die bewusste Auseinandersetzung, das Vornehmen eines Perspektivwechsels in Bezug auf die Wahrnehmung des Eigenen und des Anderen. Die Folge ist kein Verlust und keine Fragmentierung der Identität. Indem die eine Werkidentität in der Differenz zum Anderen entwickelt wird und als stabiler Faktor präsent ist, erfährt sie durch ihre situative und kontextuelle Einbettung auf einer weiteren Werkebene eine sinnhaltige Erweiterung und Komplementierung.

Im Gegensatz zur Simultanaufführung als einer primär interpretatorischen Praxis sind Poly-Werke von vornherein als Vergleichzeitigung verschiedener 
musikalischer Stränge angelegt. Zwar gilt Letzteres auch für die Werke von John Cage, der die Vergleichzeitigung seiner Werke selbst vorsah, dabei aber den konkreten Zusammengang offen ließ und nicht unbedingt spezifizierte. Die Simultanaufführung nahm einen zentralen Platz in seinem Schaffen ein. Sie war kein singuläres Experiment, sondern Teil seines Anliegens, den Formbegriff zu revidieren und gestaltete Formbeziehungen systematisch durch konstellative Beziehungen zu ersetzen. Dieser neue rezeptive Beziehungstypus ist auch für die Interpretationsästhetik späterer Poly-Werke, die das Nebeneinander der Werkstränge betonen, äußerst relevant. In Hinblick auf die Produktionsästhetik ist diese rezeptive Einstellung hingegen nicht übertragbar und markiert einen Scheideweg. Während die Simultanaufführung bei Cage auch ein Mittel war, den kompositorischen Werkbegriff beiseitezuschieben, zielt die Komposition eines Poly-Werks auf seine produktive Erweiterung und dialogische Komplementarität. Dies macht auch eine aktiv geführte Formgebung, die das Beziehungsgeflecht konkret plant und gestaltet und es nicht einfach geschehen lässt, erforderlich.

Die Erweiterung des Werks von der Einsheit zur Vielheit geht mit einer konzeptuellen Erweiterung des Polyphonie- und des Werkbegriffs einher. Sie bedeutet die Übertragung eines rein satztechnischen polyphonen Denkens auf den Werkverlauf an sich und geht mit der Ausweitung der Polysierung auf verschiedene musikalische Parameter einher. Diese Ausdehnung polyphonen Denkens auf neue Kontexte bedeutet immer auch eine Erweiterung ihres Terrains, die Zuspitzung ihrer Extreme und die Möglichkeit ihrer Umkehrung. Polyphonie muss keine Vervielfältigung im Sinne einer additiven Praxis sein. Auch die Verungleichzeitigung einer Werkentität als dissoziierende Praxis kann als Polysierung aufgefasst werden. Diese Polysierung, gleich ob additiv oder dissoziierend, stellt die Unteilbarkeit des Werks infrage, führt eine Art „dialogical turn“ für den Werkbegriff herbei. Die Teilbarkeit wiederum führt zu verschiedenen Werkidentitäten. Die Gestaltung aus der Differenz heraus scheint dabei eine notwendige Haltung zu sein, um nicht nur eine rein additive Werkschichtung herzustellen, die die Wechselwirkungen zwischen den Werken im Unbestimmten lässt, sondern die verschiedenen Werke und die Form ihrer Verknüpfung bereits im Moment ihrer Entstehung so zu antizipieren, dass alle möglichen Versionen und Entstehungsrichtungen in jeder einzelnen Version von Anfang an präsent sind. Auf diese Weise entsteht die Erweiterung und Komplementierung des Einzelnen zu einer sozialen Werkidentität bereits im individuellen Werk selbst. Die Reflexion des künstlerisches Prozesses durch die Erfahrung des Komponierens selbst lässt dabei jenen Raum im Dazwischen der Werke als einen zu gestaltenden Korridor greifbar werden, von dem aus die Beziehung der Werke zueinander bestimmt und gestaltet werden kann. 
So wird der einsinnig zu erdenkende Werkverlauf sowohl durch die Gesamtheit der weiteren Werkverläufe als auch durch ihre Beziehung untereinander komplementiert. Dies hat wiederum Konsequenzen für die Form, die sich nun in drei verschiedenen Ebenen artikuliert: erstens als innerformale Ebene, die auf das lineare Geschehen im Einzelwerk gerichtet ist; zweitens als interformale Ebene der vertikalen Beziehung zum anderen Werk; drittens als Metaform, die die verschiedenen horizontalen und vertikalen Formbeziehungen vereint.

Die verschiedenen formalen Gestaltungsmöglichkeiten wurden hier anhand eines interformalen Modells zusammengefasst, das auf die wechselseitige Beziehung der Stücke gerichtet ist. Dieses Modell ersetzt keine tiefergehende Analyse, bildet als Profil vor allem eine Art Schlüssel, um im ersten Schritt die generalisierbaren Tendenzen zu reduzieren und zu überschauen.

Auf der Basis dieses Modell war die Analyse der Formen und ihre Einordnung in homogene und heterogene Werkbildungen ein weiterer Schwerpunkt der Arbeit. Vor allem anhand der Arbeiten von Julio Estrada, Simon Steen-Andersen, Darius Milhaud und Chaya Czernowin konnten diese beiden entgegengerichteten Tendenzen sehr klar herausgearbeitet werden. Diese Modellbildung könnte dann an Grenzen stoßen, wenn es einen homogenen Ursprung im Material gibt, die Stücke aber trotzdem sehr unterschiedlich und heterogen sind. Als Orientierung ist die Unterscheidung homogen/heterogen für eine Systematisierung dennoch zentral, auch wenn man argumentieren könnte, dass es sich bei homogenen Werken nicht in gleicher Weise um PolyWerke handelt wie bei heterogenen Werken. Homogene Werke sind in erster Linie Vervielfältigungen einer Ausgangsidee. Doch gerade weil die Werke auf eine gemeinsame Wurzel zurückgehen und diese variieren oder bearbeiten, sollte bei vielen Werken die Wahrnehmung explizit auch auf die Differenzen zwischen den einzelnen Versionen gerichtet werden. Die Synthese heterogener Werke ist im Gegensatz dazu von vornherein auf eine Dialektik angelegt. Sie entsteht nicht aus der Vermehrfachung einer Idee, sondern aus unterschiedlichen Initialideen.

Es war mir wichtig, auch Werke in die Analyse einzubeziehen, in denen der Fokus der Komposition auf das freie kollektive Zusammenspiel der Musiker gerichtet ist, wie bei den Werken von Juliane Klein, Bernd Franke und Georg Friedrich Haas. Sie sind in ihrer Gestaltung in erster Linie aus der kollektiven Identität des Ensembles heraus gedacht und konzipiert. Da aber auch hier die Möglichkeit besteht, die Einzelstimmen autonom aufzuführen, erfahren sie ebenso wie das Poly-Werk eine Aufspaltung ihrer Identität in eine individuelle und eine soziale. Für ein möglichst breites Bild der Formmöglichkeiten wählte ich auch Beispiele aus, die Stücke nur in eine Richtung integrieren oder bei 
denen die Stücke nicht komplett übereinandergelegt sind, sondern sich nur für einen Teilabschnitt überlappen. Meines Erachtens handelt es sich auch hier um simultane Kontextualisierungen, die den Spagat zwischen einem Abschluss des Werkes und seiner spezifischen Öffnung für ein konkret anderes Werk verwirklicht haben. Nicht zuletzt ist eine große Bandbreite an unterschiedlichen Beispielen die Voraussetzung, um allgemeine Tendenzen in diesem inhomogenen Feld ausmachen zu können.

Es wäre zu kurz gegriffen, Simultaneität im Poly-Werk als ein einfaches Gegenmodell zur Linearität zu konstruieren. Denn die lineare Formbildung wird ja nicht aufgegeben. Sukzessivität und Simultaneität werden ebenso zusammen gedacht und müssen ebenso miteinander vereinbart werden wie die Erscheinungsformen des Gleichzeitigen und des Ungleichzeitigen. Das Poly-Werk ist der Spannung dieser verschiedenen Zeitmodi ausgesetzt. Dies spiegelt sich in der zeitlich-formalen Organisation vieler Werke in einer Ausbalancierung zwischen synchronisierten und asynchronen Prozessen wider, sei es, dass die Werke beispielsweise in ihrem Metrum, ihrem Tempo und ihrer Makroform ein gemeinsames Raster teilen, aber in ihrem mikroformalen Verläufen nicht einheitlich agieren oder umgekehrt Tempo und Metrum voneinander abweichen, die Formzäsuren jedoch einheitlich sind. Bisweilen gibt es auch verschiedene Grade von Synchronität. Die perfekte Synchronität verweist noch auf ein traditionelles Ensemblespiel, das sich in seinem zeitlichen Fortschreiten exakt synchronisiert. Daneben gibt es aber auch die imperfekte Synchronität, die die Stücke nur im Groben koordiniert und den Spielern einen zeitlichen Spielraum lässt. In vielen Werken korreliert der Grad der Synchronisation der Werke mit ihrer Materialdisposition. Tendenziell gilt: Je homogener das Material, desto einheitlicher ist die Zeitgestaltung. Je offener und heterogener das Material, desto heterogener ist auch die Organisation der Zeit.

Aus menschlicher Sicht ist der Zusammenschluss von Individuen $\mathrm{zu}$ Paaren, Gruppen oder Massen und der damit immer einhergehende Rollenund Funktionswechsel etwas ganz Natürliches. Auf der Werkebene ist das in dieser Form etwas Besonderes. Das Poly-Werk ist ein Modell, das einerseits Werkidentität als etwas Festgeschriebenes und Determiniertes anerkennt und andererseits trotzdem eine Öffnung zu anderen kollektiven Kontexten vollzieht und damit auch eine Chance zur Transformation nutzt. Für die Analyse und Deutung hätte sicher auch eine weitere Auseinandersetzung mit außermusikalischen Modellen, die oft von den Komponisten selbst für ihre jeweiligen Stücke in Anspruch genommen wurden, ein großes Potenzial. Sowohl in der eigenen künstlerischen Erfahrung, die ich im Rahmen dieser Arbeit gesammelt habe, als auch in den Gesprächen, die ich mit einigen 
Komponisten, deren Werke ich analysiert habe, führen durfte, wurde die Inspiration und Orientierung an außermusikalischen Modellen und Bildern immer wieder offenbar. Die Vielzahl an einfachen und komplexen Beispielen der Vergesellschaftung aus der Natur, der Gesellschaft und der Wissenschaft kann für zukünftige Kompositionen, Analysen, Forschungen und die Formenlehre sicher noch weiter fruchtbar gemacht werden und sollte auf keinen Fall ausgeklammert bleiben. Kürzlich bin ich so zum Beispiel auf den Dictyostelium discoideum gestoßen, einem Schleimpilz, der sowohl als Einzeller wie auch als vielzelliger Organismus existieren kann und dadurch besonders anpassungsfähig ist.

Die Simultaneität im Poly-Werk spiegelt die parallel existierenden Zeitlichkeiten unserer Welterfahrung und der Gesellschaft im räumlichen Beieinander mit ihren Parallelen, Konflikten, Gemeinsamkeiten, Vernetzungen und Ambivalenzen wider. Das Werk im Poly-Werk wird zum Ort, an dem die verschiedenen Zeitstränge akkumuliert und verknüpft werden können, zusammen- und wieder auseinandergehen, als eins und mehrere zugleich erfahrbar sind. Dieses Geschehen vollzieht sich nicht intermedial, sondern im selben Reich der Töne und Klänge. Es lässt das Konvergente sich noch näher kommen, aber auch das Divergente noch dissonanter aufeinanderprallen. 